\title{
Developing the Galactic diffuse emission model for the GLAST Large Area Telescope
}

\author{
Igor V. Moskalenko*,†, Andrew W. Strong ${ }^{* *}$, Seth W. Digel ${ }^{\ddagger} \dagger$ and Troy A. Porter ${ }^{\S}$ \\ ${ }^{*}$ Hansen Experimental Physics Laboratory, Stanford University, Stanford, CA 94305 \\ ${ }^{\dagger}$ Kavli Institute for Particle Astrophysics and Cosmology, Stanford University, Stanford, CA 94309 \\ ${ }^{* *}$ Max-Plank-Institut für extraterrestrische Physik, Postfach 1312, D-85741 Garching, Germany \\ $¥$ Stanford Linear Accelerator Center, 2575 Sand Hill Rd, Menlo Park, CA 94025 \\ ${ }^{\S}$ Santa Cruz Institute for Particle Physics, University of California, Santa Cruz, CA 95064
}

\begin{abstract}
Diffuse emission is produced in energetic cosmic ray (CR) interactions, mainly protons and electrons, with the interstellar gas and radiation field and contains the information about particle spectra in distant regions of the Galaxy. It may also contain information about exotic processes such as dark matter annihilation, black hole evaporation etc. A model of the diffuse emission is important for determination of the source positions and spectra. Calculation of the Galactic diffuse continuum $\gamma$-ray emission requires a model for CR propagation as the first step. Such a model is based on theory of particle transport in the interstellar medium as well as on many kinds of data provided by different experiments in Astrophysics and Particle and Nuclear Physics. Such data include: secondary particle and isotopic production cross sections, total interaction nuclear cross sections and lifetimes of radioactive species, gas mass calibrations and gas distribution in the Galaxy $\left(\mathrm{H}_{2}, \mathrm{H}\right.$ I, $\mathrm{H}$ II), interstellar radiation field, CR source distribution and particle spectra at the sources, magnetic field, energy losses, $\gamma$-ray and synchrotron production mechanisms, and many other issues. We are continuously improving the GALPROP model and the code to keep up with a flow of new data. Improvement in any field may affect the Galactic diffuse continuum $\gamma$-ray emission model used as a background model by the GLAST LAT instrument. Here we report about the latest improvements of the GALPROP and the diffuse emission model.
\end{abstract}

Keywords: gamma rays, cosmic rays, diffuse background, interstellar medium, gamma ray telescope PACS: $95.55 . \mathrm{Ka}, 95.85 . \mathrm{Pw}, 98.35 .-\mathrm{a}$, 98.38.-j, 98.38.Cp, 98.58.Ay, 98.70.Sa, 98.70.Vc

\section{DISCUSSION AND RESULTS}

We give a very brief summary of GALPROP; for details we refer to the relevant papers [1]-[6] and a dedicated website. The propagation equation is solved numerically on a spatial grid, either in $2 \mathrm{D}$ with cylindrical symmetry in the Galaxy or in full 3D. The boundaries of the model in radius and height, and the grid spacing, are user-definable. Parameters for all processes in the propagation equation can be controlled on input. The distribution of CR sources can be freely chosen, typically to represent supernova remnants. Source spectral shape and isotopic composition (relative to protons) are input parameters. Cross-sections are based on extensive compilations and parameterizations [7]. The numerical solution is evolved forward in time until a steady-state is reached; a time-dependent solution is also an option. Starting with the heaviest primary nucleus considered (e.g., ${ }^{64} \mathrm{Ni}$ ) the propagation solution is used to compute the source term for its spallation products, which are then propagated in turn, and so on down to protons, secondary electrons and positrons, and antiprotons. In this way secondaries, tertiaries, etc., are included. Primary electrons are treated separately. The proton, helium, and electron spectra are normalized to data; all other isotopes are determined by the source composition and propagation. $\gamma$-rays and synchrotron emission are computed using interstellar gas data (for pion-decay and bremsstrahlung) and the interstellar radiation field (ISRF) model (for inverse Compton). The computing resources required by GALPROP are moderate by current standards.

Recent extensions to GALPROP include

- new detailed calculation of the ISRF [8, 9]

- proper implementation of the anisotropic inverse Compton scattering using new ISRF (Figure 1, left)

- interstellar gas distributions based on current HI and CO surveys [10, 11]

- new parameterization of the $\pi^{0}$ production in $p p$-collisions [12] which includes the diffraction dissociation

- non-linear MHD wave - particle interactions (wave damping) [6] are included as an option

- the kinetic energy range is now extended down to $\sim 1 \mathrm{keV}$ 

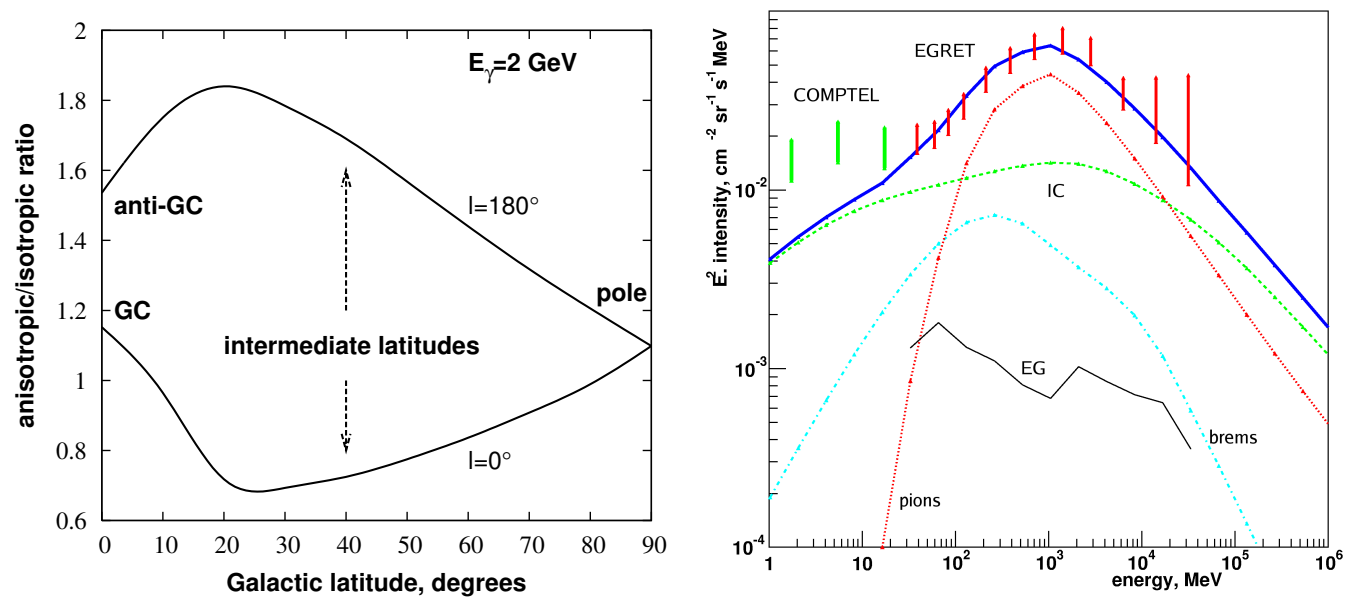

FIGURE 1. Left: The ratio of anisotropic IC to isotropic IC for Galactic longitudes $l=0^{\circ}$ and $180^{\circ}$ vs. Galactic latitude. Right: $\gamma$-ray spectrum of inner Galaxy $\left(330^{\circ}<l<30^{\circ},|b|<5^{\circ}\right)$ for an optimized model. Vertical bars: COMPTEL and EGRET data, heavy solid line: total calculated flux. This is an update of the spectrum shown in [5].

- the $\gamma$-ray calculations extend from $\mathrm{keV}$ to tens of $\mathrm{TeV}$ (e.g., Figure 1, right), and produce full sky maps as a function of energy; the output is in the FITS-format

- gas mass calibration ( $X_{\mathrm{CO}}$-factors) which can vary with position

- a dark matter package to allow for propagation of the WIMP annihilation products and calculation of the corresponding synchrotron and $\gamma$-ray skymaps

- GALPROP-DarkSUSY interface (together with T. Baltz) will become publicly available soon

- a dedicated website has been developed (http://galprop.stanford.edu)

The GALPROP code [1] was created with the following aims: (i) to enable simultaneous predictions of all relevant observations including CR nuclei, electrons and positrons, $\gamma$-rays and synchrotron radiation, (ii) to overcome the limitations of analytical and semi-analytical methods, taking advantage of advances in computing power, as CR, $\gamma$ ray and other data become more accurate, (iii) to incorporate current information on Galactic structure and source distributions, (iv) to provide a publicly-available code as a basis for further expansion. The first point is the most important: all data relating to the same system, the Galaxy, must have an internal consistency. For example, one cannot allow a model which fits secondary/primary ratios while not fitting $\gamma$-rays or not being compatible with the known interstellar gas distribution. There are many simultaneous constraints, and to find one model satisfying all of them is a challenge, which in fact has not been met up to now. Upcoming missions will benefit: GALPROP has been adopted as the standard for diffuse Galactic $\gamma$-ray emission for NASA's GLAST $\gamma$-ray observatory, and is also made use of by the ACE, AMS, HEAT and Pamela collaborations.

IVM is supported in part by NASA APRA grant, TAP is supported in part by the US Department of Energy.

\section{REFERENCES}

1. A. W. Strong, and I. V. Moskalenko, ApJ 509, 212-228 (1998).

2. I. V. Moskalenko, and A. W. Strong, ApJ 493, 694-707 (1998).

3. A. W. Strong, I. V. Moskalenko, and O. Reimer, ApJ 537, 763-784 (2000).

4. I. V. Moskalenko, A. W. Strong, J. F. Ormes, and M. S. Potgieter, ApJ 565, 280-296 (2002).

5. A. W. Strong, I. V. Moskalenko, and O. Reimer, ApJ 613, 962-976 (2004).

6. V. S. Ptuskin et al, ApJ 642, 902-916 (2006).

7. S. G. Mashnik et al., Adv. Space Res. 34, 1288-1296 (2004).

8. I. V. Moskalenko, T. A. Porter, and A. W. Strong, ApJ 640, L155-L158 (2006).

9. T. A. Porter, A. W. Strong, and S. W. Digel, in preparation (2007).

10. P. M. W. Kalberla et al., Astron. Astrophys. 440, 775-782 (2005).

11. T. M. Dame, D. Hartmann, and P. Thaddeus, ApJ 547, 792-813 (2001).

12. T. Kamae et al., ApJ 647, 692-708 (2006). 